\title{
A Study on Permanent of L-R Hexagonal Fuzzy Number Matrices
}

\section{Stephen Dinagar ${ }^{1}$ and U. Hari Narayanan ${ }^{2}$}

${ }^{1}$ PG and Research Department of Mathematics, T.B.M.L. College, Porayar-609307, India; e-mail: dsdina@rediffmail.com

${ }^{2}$ Research Scholar, PG and Research Department of Mathematics, T.B.M.L. College, Porayar-609307, India; e-mail: hari.narayanan23@gmail.com

\begin{abstract}
In this paper, it is shown that a number of properties of permanent of both square and non-square matrices present under fuzzy environment. We establish the basic formulas of determinant and permanent of matrices which contains the new properties to compare the both notions. We investigate the permanent of square L-R hexagonal fuzzy matrix (L-R HFM) using in different ways from partial derivatives. The notions of permanent of nonsquare L-R hexagonal fuzzy matrix are defined. Moreover, we derive some of the standard properties and constant matrix with the aid of above notion.
\end{abstract}

\section{Introduction}

The permanent has a rich structure when restricted to certain class of matrices, particularly, matrices of zeros and ones (entrywise) non-negative matrices and positive semi defined matrices. Furthermore, there is a certain similarity of its properties over the class of non-negative matrices and class of positive semi defined matrices. Romanwicz and Grabowski [6] used permanent of square matrix. Permanent is also used in graphtheoretic interpretations. One is, as the sum of the weights of cycle covers of a directed graph, yet another one is as the sum of weights of perfect matching in a bipartite graph.

Received: March 6, 2019; Accepted: April 20, 2019

2010 Mathematics Subject Classification: 15B15.

Keywords and phrases: L-R hexagonal fuzzy number (L-R HFN), L-R hexagonal fuzzy matrix (L-R HFM), non-square matrix, permanent, partial derivatives, constant.

Copyright (C) 2019 D. Stephen Dinagar and U. Hari Narayanan. This is an open access article distributed under the Creative Commons Attribution License, which permits unrestricted use, distribution, and reproduction in any medium, provided the original work is properly cited. 
The concept of fuzzy matrix (FM) is one of the recent developments for dealing with uncertainties present in engineering, agriculture, science, social science and also in most of our real life situations. Thomason [13] presented the article entitled convergence of power of fuzzy matrix. Ryser and Brualdi [7] studied different form of permanent of matrix. Moreover, some important results of the permanent of fuzzy matrix are established by Glynn [3]. Some of the interesting arithmetic works on fuzzy number can be found in [2]. For the first time the notion of a triangular matrix was proposed by Shyamal and Pal [8]. Recently, Das et al. [1] introduces the concept of permanent of interval valued and triangular fuzzy matrices. Stephen Dinagar and Latha [9] presented the constant type-2 TFMs. Most of our real life problems in medical sciences, engineering, management, environment and social science often involve data which are not necessarily crisp, precise, lucid and deterministic in character due to various uncertainties associated with these problems. Such uncertainties are usually being handled with the help of the topics like probability, fuzzy sets, intuitionistic fuzzy sets of fuzziness by membership function, the fuzzy number can be classified in different forms, such as triangular fuzzy number (TFN), trapezoidal fuzzy number ( $\operatorname{TrFN})$. At present, we introduce the proposed number in the article of L-R hexagonal fuzzy number (L-R HFN). Recently, Stephen Dinagar and Rajesh Kannan [10] presented the article entitled as on inventory model with allowable shortage using L-R type hexagonal fuzzy number. Recently [11, 12], we develop the concept of permanent of hexagonal fuzzy matrices.

In this article, we introduce the notion of L-R hexagonal fuzzy number (L-R HFN) in a well defined manner by conditions applied with other type of fuzzy numbers and studied in the case of permanent in both square and non-square domain of fuzzy matrices. In Section 2, we introduce L-R hexagonal fuzzy number to motivate by using of this distinct fuzzy number in real life situation. In Section 3, we have defined new L-R hexagonal fuzzy matrix (L-R HFM) and its operations. In Section 4, the definition determinant and permanent with crisp matrix and followed by the example to justify the difference of both the matrices and also have been found out a new results to compare with two matrices have been established. In Section 5, we define the permanent of square L-R hexagonal fuzzy number using in different form of partial derivatives. In Section 6, we define the permanent of non square L-R HFM and the notion of non-square constant matrix. We have also presented some of their standard properties and their proofs. In Section 7, conclusion is also included. 


\section{Hexagonal Fuzzy Number [11]}

Sometimes it may happen that some data or numbers cannot be specified precisely or accurately due to the error of the measuring technique or instruments etc. In general, the human life span can be classified by different stages. One such interesting classification is given as follows:

* Young (around (0-20))

* Early Adulthood (around (21-29))

* Middle Adulthood (around (30-40))

* Later Adulthood (around (41-60))

* Old (around (60+))

Such problems would be solved with the aid of fuzzy theory. The problem of the study of human beings whose economic and energy status are maximum, with their human life span is purely fuzzy in nature. It can be clearly noted that human beings to middle adulthood be more suit for the above said problem. This situation is clearly in nature and it can be represented by a fuzzy number called Hexagonal Fuzzy Number which is the generalization of trapezoidal fuzzy number. The advantage of this number is more than any number like Trapezoidal or Triangular. The hexagonal fuzzy number is more opt than any fuzzy numbers because of this number features do satisfies for human life span problem.

Throughout this paper, we study with the aid of L-R hexagonal fuzzy number in left and right spreads. The mathematical definition of L-R hexagonal fuzzy number is given below.

Definition 2.1. (L-R Hexagonal Fuzzy Number) [10]

An $L-R$ hexagonal fuzzy number denoted by $\tilde{A}_{h L R}=\left(m, n, \alpha_{1}, \alpha_{2}, \beta_{1}, \beta_{2}\right)_{L R}$ is a fuzzy number, where $\left(m, n, \alpha_{1}, \alpha_{2}, \beta_{1}, \beta_{2}\right)_{L R}$ are real numbers satisfying $m \leq n$, $\alpha_{1} \geq \alpha_{2}$ and $\beta_{1} \geq \beta_{2}$ and its membership function $\mu_{\tilde{A}_{h L R}}(x)$ is given by 


$$
\mu_{\tilde{A}_{h L R}}(x)= \begin{cases}0 ; & -\infty<x \leq m-\left(\alpha_{1}+\alpha_{2}\right) \\ 1-\left(\frac{m-x}{\alpha_{1}-\alpha_{2}}\right) ; & m-\left(\alpha_{1}+\alpha_{2}\right) \leq x<m-\alpha_{1} \\ 1-\frac{1}{2}\left(\frac{m-x}{\alpha_{1}}\right) ; & m-\alpha_{1} \leq x<m \\ 1 ; & m \leq x \leq n \\ 1+\frac{1}{2}\left(\frac{n-x}{\beta_{1}}\right) ; & n \leq x<n+\beta_{1} \\ 1+\frac{2}{3}\left(\frac{n-x}{\beta_{1}+\beta_{2}}\right) ; & n+\beta_{1} \leq x<n+\left(\beta_{1}+\beta_{2}\right) \\ 0 ; & x \geq n+\left(\beta_{1}+\beta_{2}\right) .\end{cases}
$$

Here the points of $m$ and $n$, with membership value of 1 , is called the flat region of mean value and $\alpha_{1}, \alpha_{2}, \beta_{1}, \beta_{2}$ are the four distinct left and right spreads of $\tilde{A}_{h L R}$, respectively.

An L-R hexagonal fuzzy number is said to be symmetric, if the sum of both its spreads are equal, i.e., if $\alpha_{1}+\alpha_{2}=\beta_{1}+\beta_{2}$ and it is denoted by $\tilde{A}_{h L R}=$ $\left(m, n, \alpha_{1}, \alpha_{2}\right)_{L R}$.

\subsection{Arithmetic operations on L-R hexagonal fuzzy numbers (HFNs)}

Here we introduce the definition of arithmetic operations between two L-R hexagonal fuzzy numbers (L-R HFNs) are given below.

Let $\tilde{A}_{h L R}=\left(m, n, \alpha_{1}, \alpha_{2}, \beta_{1}, \beta_{2}\right)_{L R}$ and $\tilde{B}_{h L R}=\left(p, q, \mu_{1}, \mu_{2}, \gamma_{1}, \gamma_{2}\right)_{L R}$ be two L-R hexagonal fuzzy numbers. Then

(i) Addition:

$$
\tilde{A}_{h L R}(+) \tilde{B}_{h L R}=\left(m+p, n+q, \alpha_{1}+\mu_{1}, \alpha_{2}+\mu_{2}, \beta_{1}+\gamma_{1}, \beta_{2}+\gamma_{2}\right)_{L R} .
$$

(ii) Subtraction:

$$
\tilde{A}_{h L R}(-) \tilde{B}_{h L R}=\left(m-p, n-q, \alpha_{1}+\mu_{1}, \alpha_{2}+\mu_{2}, \beta_{1}+\gamma_{1}, \beta_{2}+\gamma_{2}\right)_{L R} .
$$

(iii) Multiplication:

$$
\tilde{A}_{h L R}(\times) \tilde{B}_{h L R}=\left(\frac{m}{6} \sigma_{b}, \frac{n}{6} \sigma_{b}, \frac{\alpha_{1}}{6} \sigma_{b}, \frac{\alpha_{2}}{6} \sigma_{b}, \frac{\beta_{1}}{6} \sigma_{b}, \frac{\beta_{2}}{6} \sigma_{b}\right)_{L R},
$$

where $\sigma_{b}=\left(3 p+3 q-\mu_{1}-\mu_{2}+\gamma_{1}+\gamma_{2}\right)$. 
(iv) Division:

$$
\tilde{A}_{h L R}(\div) \tilde{B}_{h L R}=\left(\frac{6 m}{\sigma_{b}}, \frac{6 n}{\sigma_{b}}, \frac{6 \alpha_{1}}{\sigma_{b}}, \frac{6 \alpha_{2}}{\sigma_{b}}, \frac{6 \beta_{1}}{\sigma_{b}}, \frac{6 \beta_{2}}{\sigma_{b}}\right)_{L R},
$$

if $\sigma_{b} \neq 0$, where $\sigma_{b}=\left(3 p+3 q-\mu_{1}-\mu_{2}+\gamma_{1}+\gamma_{2}\right)$.

\section{(v) Scalar Multiplication:}

If $k \neq 0$ is scalar, then $k \tilde{A}_{h L R}$ is defined as

$$
k \tilde{A}_{h L R}= \begin{cases}\left(k m, k n, k \alpha_{1}, k \alpha_{2}, k \beta_{1}, k \beta_{2}\right) & \text { if } k \geq 0 \\ \left(k n, k m,-k \alpha_{1},-k \alpha_{2},-k \beta_{1},-k \beta_{2}\right) & \text { if } k<0 .\end{cases}
$$

\section{Definition 2.2. (Ranking Function)}

We define a ranking function $\breve{R}: F(R) \rightarrow R$ which maps each fuzzy numbers to real line $F(R)$ represented the set of all hexagonal fuzzy numbers. If $R$ be any linear ranking functions, then

$$
\breve{R}\left(\tilde{A}_{h L R}\right)=\left(\frac{3 m+3 n-\alpha_{1}-\alpha_{2}+\beta_{1}+\beta_{2}}{6}\right) .
$$

Definition 2.3. (Zero L-R Hexagonal Fuzzy Number)

If $\tilde{A}_{h L R}=(0,0,0,0,0,0)$, then $\tilde{A}_{h L R}$ is said to be zero $L-R$ hexagonal fuzzy number. It is denoted by $0_{L R}$.

Definition 2.4. (Zero-Equivalent L-R Hexagonal Fuzzy Number)

An L-R hexagonal fuzzy number $\tilde{A}_{h L R}$ is said to be zero-equivalent $L-R$ hexagonal fuzzy number if $\breve{R}\left(\tilde{A}_{h L R}\right)=0$. It is denoted by $\tilde{0}_{L R}$.

Definition 2.5. (Unit L-R Hexagonal Fuzzy Number)

If $\tilde{A}_{h L R}=(1,1,0,0,0,0)$, then $\tilde{A}_{h L R}$ is said to be unit $L-R$ hexagonal fuzzy number. It is denoted by $1_{L R}$.

Definition 2.6. (Unit-Equivalent L-R Hexagonal Fuzzy Number)

An L-R hexagonal fuzzy number $\tilde{A}_{h L R}$ is said to be unit-equivalent $L-R$ hexagonal fuzzy number if $\breve{R}\left(\tilde{A}_{h L R}\right)=1$. It is denoted by $\tilde{1}_{L R}$. 


\section{L-R Hexagonal Fuzzy Matrices (L-R HFMs)}

In this section, we propose new definitions of L-R hexagonal fuzzy number matrix and its corresponding matrix operations.

Definition 3.1. An $L-R$ hexagonal fuzzy matrix of order $m \times n$ is defined as $\hat{A}_{L R}=\left(\tilde{a}_{h L R i j}\right)_{m \times n}$, where $\left(\tilde{a}_{h L R i j}\right)=\left(m_{i j}, n_{i j}, \alpha_{1 i j}, \alpha_{2 i j}, \beta_{1 i j}, \beta_{2 i j}\right)_{L R}$ is the $i j$-th element of $\hat{A}_{L R}$, then $m_{i j}$ and $n_{i j}$ are the mean value of $\tilde{a}_{h L R i j}$ and $\alpha_{1 i j}, \alpha_{2 i j}, \beta_{1 i j}, \beta_{2 i j}$ are the left and right spreads of $\tilde{a}_{h L R i j}$, respectively.

\subsection{Operations on hexagonal fuzzy matrices (HFMs)}

Let $\hat{A}=\left(\tilde{a}_{h L R i j}\right)_{m \times n}$ and $\hat{B}=\left(\tilde{b}_{h L R i j}\right)_{m \times n}$ be two L-R HFMs of same order. Then we have the following:

1. $\hat{A}_{L R}+\hat{B}_{L R}=\left(\tilde{a}_{h L R i j}+\tilde{b}_{h L R i j}\right)$,

2. $\hat{A}_{L R}-\hat{B}_{L R}=\left(\tilde{a}_{h L R i j}-\tilde{b}_{h L R i j}\right)$,

3. For $\hat{A}_{L R}=\left(\tilde{a}_{h L R i j}\right)_{m \times n}$ and $\hat{B}=\left(\tilde{b}_{h L R i j}\right)_{n \times k}$, then $\hat{A} \hat{B}=\left(\tilde{c}_{h L R i j}\right)_{m \times k}$, where $\left(\tilde{c}_{h L R i j}\right)_{m \times k}=\sum_{p=1}^{n} \tilde{a}_{h L R i p} \tilde{b}_{h L R p j}, i=1,2, \ldots, m$ and $j=1,2, \ldots, k$,

4. $\hat{A}_{L R}^{T}$ or $\hat{A}_{L R}^{\prime}=\left(\tilde{a}_{h L R j i}\right)$,

5. $k \hat{A}_{L R}=\left(k \tilde{a}_{h L R i j}\right)$, where $k$ is scalar.

We now define some special types of L-R HFMs corresponding to special classical matrices.

Definition 3.2. (Zero L-R Hexagonal Fuzzy Matrix)

An L-R hexagonal fuzzy matrix (L-R HFM) is said to be a zero $L-R H F M$ if all its entries are $0_{L R}$ and it is denoted by $\hat{O}_{L R}$.

\section{Definition 3.3. (Unit L-R Hexagonal Fuzzy Matrix)}

The square L-R HFM is said to be a unit L-R HFM if the diagonal elements are $1_{L R}$ and the rest of elements are $0_{L R}$, i.e., if $\tilde{a}_{h L R i j}=(1,1,0,0,0,0)_{L R}$ and $\tilde{a}_{h L R i j}=$ $(0,0,0,0,0,0)_{L R}, i \neq j$ for all $i, j$. It is denoted by $\hat{I}_{L R}$. 
Definition 3.4. A square L-R HFM $\hat{A}_{L R}=\left(\tilde{a}_{h L R i j}\right)$ is said to be symmetric if $\hat{A}_{L R}=\hat{A}_{L R}^{\prime}$, i.e., if $\left(\tilde{a}_{h L R i j}\right)=\left(\tilde{a}_{h L R j i}\right)$ for all $i, j$.

Definition 3.5. A square L-R HFM $\hat{A}_{L R}=\left(\tilde{a}_{h L R i j}\right)=0_{L R}$, i.e., if $\left(\tilde{a}_{h L R i j}\right)=$ $-\left(\tilde{a}_{h L R i j}\right)$ for all $i, j$ and $\left(\tilde{a}_{h L R i i}\right)=0_{L R}$.

\section{A Comparison between Permanent and Determinant of Matrices}

In this section, we have to compare both the permanent and determinant of matrices using crisp matrix and find out the new results are justified.

\section{Definition 4.1. (Determinant)}

Let $A=\left(a_{i j}\right)_{n \times n}$ be a crisp matrix of order $n \times n$. Then the determinant of $A$ is denoted by $\operatorname{det}(A)$ or $|A|$ and defined as

$$
|A|=\sum_{\sigma \in s_{n}} \operatorname{sgn} \sigma \prod_{i=1}^{n} a_{i} \sigma(i),
$$

where $s_{n}$ denotes the symmetric group of all permutations of the indices $\{1,2, \ldots, n\}$ and $\operatorname{sgn} \sigma$ is +1 for even permutations and -1 for odd permutations.

\section{Definition 4.2. (Permanent)}

If $A=\left(a_{i j}\right)_{n \times n}$ is a crisp matrix of order $n \times n$, then the permanent of $A$ is denoted by $\operatorname{per}(A)$ and defined as

$$
\operatorname{per}(A)=\sum_{\sigma \in s_{n}} \prod_{i=1}^{n} a_{i} \sigma(i),
$$

where $s_{n}$ denotes the symmetric group of order $n$.

The definition of permanent [4] is similar to the definition of determinant except the sign of each term in summation. The number of terms over summation are both cases but the sign associated in each term are all positive in case of permanent. The permanent cannot compete with determinant, in terms of the depth of theory and breadth of applications, but it is safe to say that the permanent also exhibits both these characteristic in ample measure, a fact that has not receive enough attention. 
Here three ways to calculate $\operatorname{per}(A)$ for general $3 \times 3$ matrix for $A=\left(\begin{array}{lll}a & d & g \\ b & e & h \\ c & f & i\end{array}\right)$.

The classical formula using all the permutation in $S_{3}$ is

$$
\operatorname{Per}(A)=a e i+b f g+c d h+a f h+b d i+c e g
$$

Ryser's [7] method gives

$$
\begin{aligned}
\operatorname{Per}(A)= & (a+b+c)(d+e+f)(g+h+i)-(a+b \cdot(d+e)(g+h) \\
& -(a+c)(d+f)(g+i)-(b+c)(e+f)(h+i)+a d g+b e f+c f i .
\end{aligned}
$$

Glynn [3] method gives

$$
\begin{aligned}
2^{2} \operatorname{Per}(A)= & (a+b+c)(d+e+f)(g+h+i)-(a-b+c)(d-e+f)(g-h+i) \\
& -(a+b-c)(d+e-f)(g+h-i)+(a-b-c)(d-e-f)(g-h-i) .
\end{aligned}
$$

Compare with all the three formulas, we take the first formula to apply all problems throughout this paper and following an example is,

Let us consider an example to illustrate both the determinant and permanent of crisp matrix.

$$
\begin{aligned}
& \text { Example 4.3. Let } A=\left[\begin{array}{lll}
1 & 2 & 4 \\
3 & 5 & 7 \\
2 & 1 & 6
\end{array}\right] \text {. Then } \\
& \qquad \begin{aligned}
|A| & =1.5 .6-1.7 .1-2.3 .6+2.2 .7+4.3 .1-4.5 .2 \\
|A| & =-13
\end{aligned}
\end{aligned}
$$

and

$$
\begin{aligned}
& \operatorname{per}(A)=1.5 .6+1.7 .1+2.3 .6+2.2 .7+4.3 .1+4.5 .2 \\
& \operatorname{per}(A)=153 .
\end{aligned}
$$

\subsection{Special properties of permanent}

Properties of permanent are also presented below. 
1. For a crisp matrix $A=\left(a_{i j}\right)_{n \times n}$ is the positive square matrix of order $n$, then $\operatorname{per}(A) \geq \operatorname{det}(A)$.

2. For a crisp matrix $A=-\left(a_{i j}\right)_{n \times n}$, then

- If $A=-\left(a_{i j}\right)_{n \times n}$ is even square matrix of order $n$, then $\operatorname{per}(A) \geq \operatorname{det}(A)$.

- If $B=-\left(b_{i j}\right)_{n \times n}$ is odd square matrix of order $n$, then $\operatorname{per}(B) \leq \operatorname{det}(B)$.

3. If any one of the row (or) column of a crisp matrix $A=\left(a_{i j}\right)_{n \times n}$ of order $n$ is negative, then the permanent of a matrix is negative.

4. If any one of the row (or) column of a crisp matrix $A=\left(a_{i j}\right)_{n \times n}$ of order $n$ is negative, then $\operatorname{per}(A) \leq \operatorname{det}(A)$.

\section{Verifications:}

The above said special properties of permanent have been verified by the counter examples.

Example 4.4. Let $A=\left[\begin{array}{ll}1 & 2 \\ 3 & 4\end{array}\right]$. Then

$$
\begin{aligned}
& \operatorname{per}(A)=10, \\
& \operatorname{det}(A)=-2 .
\end{aligned}
$$

From (4.1) and (4.2),

$$
\operatorname{per}(A)>\operatorname{det}(A)
$$

In particular, $\operatorname{per}(A)=\operatorname{det}(A)$ whenever zero matrix and triangular matrix perform.

Therefore, $\operatorname{per}(A) \geq \operatorname{det}(A)$.

Example 4.5. (i) Let $A=\left[\begin{array}{ll}-1 & -2 \\ -3 & -4\end{array}\right]_{2 \times 2}$. Then

$$
\begin{aligned}
& \operatorname{per}(A)=10, \\
& \operatorname{det}(A)=-2 .
\end{aligned}
$$


From (4.3) and (4.4),

$$
\operatorname{per}(A)>\operatorname{det}(A)
$$

In particular, matrix will become zero matrix and triangular matrix, then $\operatorname{per}(A)=\operatorname{det}(A)$.

Therefore, $\operatorname{per}(A) \geq \operatorname{det}(A)$.

(ii) Let $B=\left[\begin{array}{ccc}-1 & -2 & -1 \\ 0 & -3 & -2 \\ -5 & -3 & -2\end{array}\right]_{3 \times 3}$. Then

$$
\begin{gathered}
\operatorname{per}(A)=-47 \\
\operatorname{det}(A)=-5
\end{gathered}
$$

From (4.5) and (4.6),

$$
\operatorname{per}(A)<\operatorname{det}(A)
$$

In particular, matrix will become zero matrix and triangular matrix, then $\operatorname{per}(B)=\operatorname{det}(B)$.

Therefore, $\operatorname{per}(B) \leq \operatorname{det}(B)$.

$$
\begin{aligned}
& \text { Example 4.6. Let } A=\left[\begin{array}{ccc}
-1 & -2 & -1 \\
0 & 3 & 2 \\
5 & 3 & 2
\end{array}\right] \text {. Then } \\
& \operatorname{per}(A)=-1.3 .2+-1.3 .2+-2.0 .2+-2.5 .2+-1.0 .3+-1.5 .3 . \\
& \operatorname{per}(A)=-6-6+0-20+0-15 . \\
& \operatorname{per}(A)=-47 .
\end{aligned}
$$

Therefore, the permanent of a matrix is negative.

$$
\text { Example 4.7. Let } A=\left[\begin{array}{ccc}
-1 & -2 & -1 \\
0 & 3 & 2 \\
5 & 3 & 2
\end{array}\right] \text {. Then }
$$




$$
\begin{gathered}
\operatorname{per}(A)=-47 \\
\operatorname{det}(A)=-5
\end{gathered}
$$

From (4.7) and (4.8),

$$
\operatorname{per}(A)<\operatorname{det}(A)
$$

In particular, matrix will become zero matrix and triangular matrix, then $\operatorname{per}(A)=\operatorname{det}(A)$.

Therefore, $\operatorname{per}(A) \leq \operatorname{det}(A)$.

\section{Permanent of Square L-R Hexagonal Fuzzy Matrices}

In this section, we investigate the permanent of L-R hexagonal fuzzy matrix involving partial derivatives of homogeneous polynomial of degree $n$ and characteristic $p$ over a fuzzy field $\mathbb{F}$.

\subsection{Permanent with partial derivatives}

Let $\hat{A}_{L R}$ be an $n \times n$ L-R hexagonal fuzzy matrix defined as

$$
\tilde{A}_{h L R}=\prod_{i=1}^{n} \hat{A}_{i L R}
$$

where $\hat{A}_{i L R}=\sum_{j=1}^{n}\left(\tilde{a}_{h i j} \cdot \tilde{x}_{h j}\right)_{L R}, i=1,2, \ldots, n$.

Since $\hat{A}_{L R}$ is an homogeneous polynomial L-R hexagonal fuzzy number of degree $n$ in $\left(\tilde{x}_{h 1}, \ldots, \tilde{x}_{h n}\right)$, we can write

$$
\tilde{A}_{h L R}=\sum_{\alpha_{1} \cdots \alpha_{n}}\left(\tilde{x}_{h \alpha_{1} \cdots \alpha_{n}}\right)_{L R} \frac{\tilde{x}_{h L R 1}^{\alpha_{1}}}{\alpha_{1} !} \cdot \frac{\tilde{x}_{h L R 2}^{\alpha_{2}}}{\alpha_{2} !} \cdots \frac{\tilde{x}_{h L R n}^{\alpha_{n}}}{\alpha_{n} !},
$$

where the summation is over all sequence $\left(\alpha_{1} \cdots \alpha_{n}\right)$ of non-negative integer satisfying $\alpha_{1}+\cdots+\alpha_{n}=n$. The coefficient $\left(\tilde{x}_{h \alpha_{1} \cdots \alpha_{n}}\right)_{L R}$ is equal to the partial derivatives form, i.e.,

$$
\frac{\partial^{n} \cdot \tilde{A}_{h L R}}{\partial \tilde{x}_{h L R j_{1}} \cdots \partial \tilde{x}_{h L R j_{n}}}, \quad\left(j_{1}, j_{2}, \ldots, j_{n}\right)=\left(\tilde{S}_{h \alpha_{1} \cdots \alpha_{n}}\right)_{L R}
$$


Then the derivative is

$$
\begin{aligned}
\frac{\partial^{n} \cdot \tilde{A}_{h L R}}{\partial \tilde{x}_{h L R j_{1}} \cdots \partial \tilde{x}_{h L R j_{n}}} & =\frac{\partial^{\alpha_{1}+\alpha_{2}+\cdots+\alpha_{n}} \cdot \tilde{A}_{h L R}}{\partial \tilde{x}_{h L R 1}^{\alpha_{1}} \cdots \partial \tilde{x}_{h L R n}^{\alpha_{n}}} \\
& =\frac{\partial^{\alpha_{1}+\alpha_{2}+\cdots+\alpha_{n}}}{\partial \tilde{x}_{h L R 1}^{\alpha_{1}} \cdots \partial \tilde{x}_{h L R n}^{\alpha_{n}}}\left(\tilde{x}_{h \alpha_{1} \cdots \alpha_{n}}\right)_{L R} \frac{\tilde{x}_{h L R 1}^{\alpha_{1}}}{\alpha_{1} !} \cdot \frac{\tilde{x}_{h L R 2}^{\alpha_{2}} \cdots \frac{\tilde{x}_{h L R n}^{\alpha_{n}}}{\alpha_{2} !}}{\alpha_{n} !} \\
& =\left(\tilde{x}_{h \alpha_{1} \cdots \alpha_{n}}\right)_{L R}\left(\frac{1}{\alpha_{1} !} \frac{\partial^{\alpha_{1}} \tilde{x}_{h L R 1}^{\alpha_{1}}}{\partial \tilde{x}_{h L R 1}^{\alpha_{1}}}\right) \cdots\left(\frac{1}{\alpha_{1} !} \frac{\partial^{\alpha_{n}} \tilde{x}_{h L R n}^{\alpha_{n}}}{\partial \tilde{x}_{h L R n}^{\alpha_{n}}}\right) \\
& =\left(\tilde{x}_{h \alpha_{1} \cdots \alpha_{n}}\right)_{L R}\left(\frac{\alpha_{1} !}{\alpha_{1} !} \frac{\alpha_{1} !}{\alpha_{1} !}\right) \\
\frac{\partial^{n} \cdot \tilde{A}_{h L R}}{\partial \tilde{x}_{h L R j_{1}} \cdots \partial \tilde{x}_{h L R j_{n}}} & =\left(\tilde{x}_{h \alpha_{1} \cdots \alpha_{n}}\right)_{L R} .
\end{aligned}
$$

Now, we compute $\frac{\partial^{n} \tilde{A}_{h L R}}{\partial \tilde{x}_{h L R j_{i}}}, \quad j_{i}=\left(\tilde{S}_{h \alpha_{i}}\right)_{L R} \forall i=1,2, \ldots, n$.

Now the result is

$$
j_{i}=\left(\tilde{S}_{h \alpha_{i}}\right)_{L R}=\left(\tilde{S}_{h \alpha_{1}}\right)_{L R} \forall i=1,2, \ldots, n .
$$

Since $\tilde{A}_{i L R}$ is a linear function of $\tilde{x}_{h L R j_{i}}^{\prime} s$, then we have

$$
\frac{\partial^{2} \hat{A}_{i L R}}{\partial \tilde{x}_{h L R k} \cdot \partial \tilde{x}_{h L R k}}=0
$$

for any $k$ and $l$ are defined by differentiation

$$
\begin{aligned}
\frac{\partial^{n} \cdot \tilde{A}_{h L R}}{\partial \tilde{x}_{h L R j_{1}} \cdots \partial \tilde{x}_{h L R j_{n}}} & =\frac{\partial^{n} \cdot \tilde{A}_{h L R}}{\partial \tilde{x}_{h L R 1}, \partial \tilde{x}_{h L R 2}, \ldots, \partial \tilde{x}_{h L R n}} \\
& =\sum_{\sigma \in s_{n}} \frac{\partial \tilde{A}_{h L R \sigma(1)}}{\partial \tilde{x}_{h L R 1}} \cdot \frac{\partial \tilde{A}_{h L R \sigma(2)}}{\partial \tilde{x}_{h L R 2}} \cdots \frac{\partial \tilde{A}_{h L R \sigma(n)}}{\partial \tilde{x}_{h L R n}}
\end{aligned}
$$




$$
\begin{aligned}
& =\sum_{\sigma \in s_{n}} \prod_{i=1}^{n} \frac{\partial \tilde{A}_{h L R \sigma(i)}}{\partial \tilde{x}_{i h L R}} \\
& =\sum_{\sigma \in s_{n}} \prod_{i=1}^{n}\left(\tilde{a}_{h \sigma(i) i}\right)_{L R},
\end{aligned}
$$

where $\frac{\partial \tilde{A}_{h L R \sigma(i)}}{\partial \tilde{x}_{i h L R}}=\left(\tilde{a}_{h \sigma(j) j}\right)_{L R}=\operatorname{per}\left(\hat{A}_{L R}\right)$. Therefore,

$$
\frac{\partial^{n} \cdot \tilde{A}_{h L R}}{\partial \tilde{x}_{h L R j_{1}} \cdots \partial \tilde{x}_{h L R j_{n}}}=\operatorname{per}\left(\hat{A}_{L R}\right)
$$

Let now $\left(j_{1}, j_{2}, \ldots, j_{n}\right)=\left(\tilde{S}_{h \alpha_{1} \cdots \alpha_{n}}\right)_{L R}$. Again by differentiation and recalling equation (5.2), we have

$$
\begin{aligned}
\frac{\partial^{n} \cdot \tilde{A}_{h L R}}{\partial \tilde{x}_{h L R j_{1}} \cdots \partial \tilde{x}_{h L R j_{n}}} & =\sum_{\sigma \in s_{n}} \prod_{i=1}^{n} \frac{\partial \tilde{A}_{h L R \sigma(i)}}{\partial \tilde{x}_{h L R j_{i}}} \\
& =\sum_{\sigma \in s_{n}} \prod_{i=1}^{n}\left(\tilde{a}_{h \sigma(i) j_{i}}\right)_{L R} \\
\frac{\partial^{n} \cdot \tilde{A}_{h L R}}{\partial \tilde{x}_{h L R j_{1}} \cdots \partial \tilde{x}_{h L R j_{n}}} & =\operatorname{per}\left(\hat{A}_{L R}\left(\alpha_{1} \cdots \alpha_{n}\right)\right) .
\end{aligned}
$$

\subsection{Permanent of $L-R$ hexagonal fuzzy number from partial derivatives}

Definition 5.1. Let $\hat{A}_{L R}=\left(\tilde{a}_{h i j}\right)_{L R}$ be an $n \times n$ L-R HFM over a fuzzy field $\mathbb{F}$ of characteristic $p$. Then it is defined as

$$
\operatorname{per}\left(\hat{A}_{L R}\right)=(-1)^{n}\left(\frac{\partial^{n}}{\partial \tilde{a}_{h L R 11} \cdots \partial \tilde{a}_{h L R n n}}\right)^{p-2} \operatorname{det}\left(\hat{A}_{L R}\right)^{p-1} .
$$

Theorem 5.2. Let $\hat{A}_{L R}=\left(\tilde{a}_{h i j}\right)_{L R}$ be an $n \times n$ L-R HFM over a fuzzy field of characteristic $p$. Then 


$$
\operatorname{per}\left(\hat{A}_{L R}\right)=(-1)^{n}\left(\frac{\partial^{n}}{\partial \tilde{a}_{h L R 11} \cdots \partial \tilde{a}_{h L R n n}}\right)^{p-2} \operatorname{det}\left(\hat{A}_{L R}\right)^{p-1}
$$

Proof. The polynomial $f: \operatorname{det}\left(\tilde{A}_{L R}\right)^{p-1}$ is homogeneous of degree $n(p-1)$ in the $n^{2}$ variables $\left(\tilde{a}_{h L R i j}\right)$. To obtain a non-zero monomial in these variables from the operator

$$
\left(\frac{\partial^{n}}{\partial \tilde{a}_{h L R 11} \cdots \partial \tilde{a}_{h L R n n}}\right)^{p-2}=\prod_{i=1}^{n}\left(\frac{\partial^{p-2}}{\partial \tilde{a}_{h L R i i}^{p-2}}\right)
$$

that acts upon $f$. Now, we consider the monomial in $f$ are of the form

$$
\hat{Z}_{L R}=\prod_{i=1}^{n} \tilde{a}_{h L R i i}^{p-2} \cdot k,
$$

where $k$ is a homogeneous polynomial of degree $n$. Since $\operatorname{det}\left(\tilde{A}_{L R}\right)$ is the sum of the products of the form

$$
\prod_{i=1}^{n} \tilde{a}_{h L R i q(i)}
$$

It is clear that $k$ must be a similar form corresponding to the unique permutation $q \in s_{n}$. Let $q$ have $r$ even cycle of size $a_{1} \cdots a_{r}\left(a_{i} \geq 2\right), s$ odd cycle of size $b_{1} \cdots b_{r}\left(b_{i} \geq 3\right)$ and $c$ cycle of size is fixed point of one. Then we note that $n \equiv s+c(\bmod 2)$, since the even cycles can be neglected when we calculate the length of permutation mod 2. Also, $q$ is an even permutation if and only if $r$ is even. When $k$ is produced by the above partial differentiation from $\hat{Z}_{L R}$, there is a factor of $((p-2) !)^{r+s} \cdot((p-1) !)^{c} \equiv(-1)^{c}$ in general function of $p$. This is because a cycle of size one in $q$ corresponds to an $\tilde{a}_{h L R i i}^{p-2}$ in $\hat{Z}_{L R}$ and differentiates $p-2$ times to $(p-1) ! \tilde{a}_{h L R i i}$, otherwise $\tilde{a}_{h L R i i}^{p-2} \cdot \tilde{a}_{h L R j k}$ differentiates to $(p-2) ! \tilde{a}_{h L R j k}$ and $(p-2) ! \equiv 1$ while $(p-1) ! \equiv-1(\bmod p)$. Next, each function from $\{1,2, \ldots, r+s\} \rightarrow\{1,2, \ldots, p-1\}$ corresponds to a way that $\hat{Z}_{L R}$ can appear as a polynomial in the product of $\operatorname{det}\left(\hat{A}_{L R}\right),(p-1)$ times. This is because the non-diagonal $\tilde{a}_{h} L R i j{ }^{\prime} s$ of $k$ in each non-trivial cycle must be assigned to one of the 
$p-1$ permutation of the $\hat{A}_{L} R^{\prime} s$. The number of these function is $(p-1)^{r+1} \equiv(-1)^{r+s}$ in general function of $p$. In addition each of these ways of producing $k$ gives the same sign as $q$, since the $p-1$ permutations from the determinants have a product that is $q$ and so the signs multiply to give $\operatorname{sgn}(q)=(-1)^{r}$.

Hence the total coefficient of $k$ in the partial derivative of $\hat{A}_{L R}$ and hence of $\operatorname{det}\left(\hat{A}_{L R}\right)^{p-1}$ is $(-1)^{r} \cdot(-1)^{c} \cdot(-1)^{r+s} \equiv(-1)^{s+c} \equiv(-1)^{n}(\bmod p)$. Thus the formula in the theorem is true.

Corollary 5.3. Let $\hat{A}_{L R}=\left(\tilde{a}_{h i j}\right)_{L R}$ be an $n \times n$ L-R HFM over a fuzzy field $\mathbb{F}$ characteristic three. Then

$$
\operatorname{per}\left(\hat{A}_{L R}\right)=(-1)^{n}\left(\frac{\partial^{n}}{\partial \tilde{a}_{h L R 11} \cdots \partial \tilde{a}_{h L R n n}}\right) \operatorname{det}\left(\hat{A}_{L R}\right)^{2} .
$$

Example 5.4. Let $\hat{A}_{L R}=\left(\begin{array}{cc}\tilde{a}_{h L R} & \tilde{b}_{h L R} \\ \tilde{c}_{h L R} & \tilde{d}_{h L R}\end{array}\right)$. Then

$$
\begin{aligned}
\operatorname{per}\left(\hat{A}_{L R}\right) & =\left(\begin{array}{ll}
\tilde{a}_{h L R} & \tilde{b}_{h L R} \\
\tilde{c}_{h L R} & \tilde{d}_{h L R}
\end{array}\right) \\
& =(-1)^{2}\left(\frac{\partial^{2}}{\partial \tilde{a}_{h L R} \cdot \partial \tilde{d}_{h L R}}\right) \operatorname{det}\left(\left(\begin{array}{cc}
\tilde{a}_{h L R} & \tilde{b}_{h L R} \\
\tilde{c}_{h L R} & \tilde{d}_{h L R}
\end{array}\right)\right)^{2} \\
& =\left(\frac{\partial^{2}}{\partial \tilde{a}_{h L R} \cdot \partial \tilde{d}_{h L R}}\right)\left(\tilde{a}_{h L R} \cdot \tilde{d}_{h L R}-\tilde{b}_{h L R} \cdot \tilde{c}_{h L R}\right) \\
& =\left(\frac{\partial}{\partial \tilde{a}_{h L R}}\right)\left[2\left(\tilde{a}_{h L R} \cdot \tilde{d}_{h L R}-\tilde{b}_{h L R} \cdot \tilde{c}_{h L R}\right) \cdot \tilde{a}_{h L R}\right] \\
& =2\left(\tilde{a}_{h L R} \cdot \tilde{d}_{h L R}-\tilde{b}_{h L R} \cdot \tilde{c}_{h L R}\right)+2 \tilde{a}_{h L R} \cdot \tilde{d}_{h L R} \\
& =\tilde{a}_{h L R} \cdot \tilde{d}_{h L R}+\tilde{b}_{h L R} \cdot \tilde{c}_{h L R}(\bmod 3)(\text { by Theorem 5.2) } \\
\operatorname{per}\left(\hat{A}_{L R}\right) & =\tilde{a}_{h L R} \cdot \tilde{d}_{h L R}+\tilde{b}_{h L R} \cdot \tilde{c}_{h L R} .
\end{aligned}
$$


Theorem 5.5. Let $\hat{A}_{L R}=\left(\tilde{a}_{h i j}\right)_{L R}$ be an $n \times n$ L-R HFM over a fuzzy field $\mathbb{F}$ characteristic three. Then

$$
\operatorname{per}\left(\hat{A}_{L R}\right)=(-1)^{n-1} \sum_{X \subset\{2, \ldots, n\}} \operatorname{det}\left(\hat{A}_{L R x}\right) \operatorname{det}\left(\hat{A}_{L R \bar{x}}\right),
$$

where $\hat{A}_{L R x}$ is the principal submatrix of $\hat{A}_{L R}$ induced by the rows and columns of $\hat{A}_{L R}$ indexed by $x$ and $\bar{x}$ is the complement of $x$ in $\{1,2, \ldots, n\}$.

Proof. For $i, j, k \in\{1,2, \ldots, n\}$ defined $\hat{A}_{L R}$ where $i, j, k$ is said to be principal submartix of $\hat{A}_{L R}$ induced by removing the rows and columns indexed by $i, j, k$.

From the corollary,

$$
\begin{aligned}
\operatorname{per}\left(\hat{A}_{L R}\right)= & (-1)^{n}\left(\prod_{i=1}^{n} \frac{\partial}{\partial \tilde{a}_{h L R i i}}\right) \operatorname{det}\left(\hat{A}_{L R}\right)^{2} \\
= & (-1)^{n}\left(\prod_{i=2}^{n} \frac{\partial}{\partial \tilde{a}_{h L R i i}}\right) \frac{\partial}{\partial \tilde{a}_{h L R 11}} \operatorname{det}\left(\hat{A}_{L R}\right) \\
= & (-1)^{n}\left(\prod_{i=2}^{n} \frac{\partial}{\partial \tilde{a}_{h L R i i}}\right) 2\left(\left|\hat{A}_{L R}\right| \cdot\left|\hat{A}_{L R 1}\right|\right) \\
= & (-1)^{n}\left(\prod_{i=3}^{n} \frac{\partial}{\partial \tilde{a}_{h L R i i}}\right) \frac{\partial}{\partial \tilde{a}_{h L R 22}}\left(\left|\hat{A}_{L R}\right| \cdot\left|\hat{A}_{L R 1}\right|\right) \\
= & (-1)^{n}\left(\prod_{i=3}^{n} \frac{\partial}{\partial \tilde{a}_{h L R i i}}\right)\left|\hat{A}_{L R 2}\right| \cdot\left|\hat{A}_{L R 1}\right|+\left|\hat{A}_{L R}\right| \cdot\left|\hat{A}_{L R 1,2}\right| \\
= & (-1)^{n}\left(\prod_{i=4}^{n} \frac{\partial}{\partial \tilde{a}_{h L R i i}}\right) \frac{\partial}{\partial \tilde{a}_{h L R 33}}\left(\left|\hat{A}_{L R 2}\right| \cdot\left|\hat{A}_{L R 1}\right|+\left|\hat{A}_{L R}\right| \cdot\left|\hat{A}_{L R 1,2}\right|\right) \\
= & (-1)^{n}\left(\prod_{L R 1,2}^{n} \frac{\partial}{\partial \tilde{a}_{h L R i i}}\right)\left|\hat{A}_{L R 2,3}\right| \cdot\left|\hat{A}_{L R 1}\right|+\left|\hat{A}_{L R 1,3}\right| \cdot\left|\hat{A}_{L R 2}\right|+\left|\hat{A}_{L R}\right| \cdot\left|\hat{A}_{L R 1,2,3}\right|
\end{aligned}
$$




$$
\operatorname{per}\left(\hat{A}_{L R}\right)=(-1)^{n} \sum_{X \subset\{1,2, \ldots, m\}}\left|\hat{A}_{L R x}\right| \cdot\left|\hat{A}_{L R \bar{x}}\right|
$$

Hence the proof.

\section{Permanent of Non-Square L-R Hexagonal Fuzzy Matrices}

In this section, we introduce the notion of permanent of L-R HFM to define the order $m \times n$. Also, we define the notion of constant L-R HFM in the same order and its relevant properties are discussed.

Definition 6.1. Let $\hat{A}_{L R}=\left(\tilde{a}_{h L R i j}\right)_{m \times n}$ be a non-square L-R HFM of order $m \times n$ where $\tilde{a}_{h L R i j}=\left(m_{i j}, n_{i j}, \alpha_{1 i j}, \alpha_{2 i j}, \beta_{1 i j}, \beta_{2 i j}\right)_{L R}$ be an L-R hexagonal fuzzy number. Then the permanent of $\hat{A}_{L R}$ is denoted by $\operatorname{per}\left(\hat{A}_{L R}\right)$ and is defined by

$$
\begin{aligned}
\operatorname{per}\left(\hat{A}_{L R}\right)= & \sum_{q \in S} \prod_{i=1}^{m} \tilde{a}_{h L R i q(i)} \text { for } m \leq n \\
& \quad[\text { where } s \text { is the set of all one-to-one mapping } \\
& \quad \text { rom }\{1,2, \ldots, m\} \text { to }\{1,2, \ldots, n\}] \\
= & \sum_{q \in S} \prod_{j=1}^{m} \tilde{a}_{h L R q(j) j} \text { for } m>n
\end{aligned}
$$

[where $s$ is the set of all one-to-one mapping from $\{1,2, \ldots, n\}$ to $\{1,2, \ldots, m\}]$

Two expressions are written for the permanent of matrix, because for $m>n$, there are one-to-one mapping $\{1,2, \ldots, n\}$ to $\{1,2, \ldots, m\}$. In this case, no one-to-one is possible from $\{1,2, \ldots, m\}$ to $\{1,2, \ldots, n\}$. But for $m \leq n$ the one-to-one mapping are possible from $\{1,2, \ldots, m\}$ to $\{1,2, \ldots, n\}$.

\subsection{Properties of non-square permanent of HFMs}

Property 6.2. Fundamental Properties of Permanent of Non-Square L-R HFMs

For any two non-square L-R HFMs $\hat{A}_{L R}$ and $\hat{B}_{L R}$ of order $m \times n$, then 
(i) $\operatorname{per}\left(\hat{A}_{L R}+\hat{B}_{L R}\right) \geq \operatorname{per}\left(\hat{A}_{L R}\right)+\operatorname{per}\left(\hat{B}_{L R}\right)$.

(ii) $\operatorname{per}\left(\hat{A}_{L R}-\hat{B}_{L R}\right) \leq \operatorname{per}\left(\hat{A}_{L R}\right)-\operatorname{per}\left(\hat{B}_{L R}\right)$.

(iii) $\operatorname{per}\left(\hat{A}_{L R} \hat{B}_{L R}^{T}\right) \geq \operatorname{per}\left(\hat{A}_{L R}\right) \cdot \operatorname{per}\left(\hat{B}_{L R}^{T}\right)$.

Proof. The proof of (i), (ii), and (iii) are obvious from the fundamental theorem.

Property 6.3. For any two non-square L-R HFMs $\hat{A}_{L R}$ and $\hat{B}_{L R}$ of same order such that $\hat{A}_{L R} \leq \hat{B}_{L R} \Rightarrow \operatorname{per}\left(\hat{A}_{L R}\right) \leq \operatorname{per}\left(\hat{B}_{L R}\right)$.

Proof. Let $\hat{A}_{L R}=\left(\tilde{a}_{h L R i j}\right)_{m \times n}$ and $\hat{B}_{L R}=\left(\tilde{a}_{h L R i j}\right)_{m \times n}$ be two non-square L-R HFMs, where $\tilde{a}_{h L R i j}=\left(m_{i j}, n_{i j}, \alpha_{1 i j}, \alpha_{2 i j}, \beta_{1 i j}, \beta_{2 i j}\right)_{L R}$ and $\tilde{b}_{h L R i j}=\left(p_{i j}, q_{i j}, \mu_{1 i j}\right.$, $\left.\mu_{2 i j}, \gamma_{1 i j}, \gamma_{2 i j}\right)_{L R}$. Then, $\hat{A}_{L R} \leq \hat{B}_{L R} \Rightarrow \tilde{a}_{h L R i j} \leq \tilde{b}_{h L R i j} \Rightarrow\left(m_{i j}, n_{i j}, \alpha_{1 i j}, \alpha_{2 i j}, \beta_{1 i j}\right.$, $\left.\beta_{2 i j}\right)_{L R} \leq\left(p_{i j}, q_{i j}, \mu_{1 i j}, \mu_{2 i j}, \gamma_{1 i j}, \gamma_{2 i j}\right)_{L R}$.

When $m \leq n$,

$$
\begin{aligned}
\operatorname{per}\left(\hat{A}_{L R}\right) & =\sum_{q \in S} \prod_{i=1}^{m} \tilde{a}_{h L R i q(i)} \\
& =\sum_{q \in S} \prod_{i=1}^{m}\left(m_{i q(i)}, n_{i q(i)}, \alpha_{1 i q(i)}, \alpha_{2 i q(i)}, \beta_{1 i q(i)}, \beta_{2 i q(i)}\right)_{L R} \\
& \leq \sum_{q \in S} \prod_{i=1}^{m}\left(p_{i q(i)}, q_{i q(i)}, \mu_{1 i q(i)}, \mu_{2 i q(i)}, \gamma_{1 i q(i)}, \gamma_{2 i q(i)}\right)_{L R} \\
& \leq \sum_{q \in S} \prod_{i=1}^{m} \tilde{b}_{h L R i q(i)} \\
& \leq \operatorname{per}\left(\hat{B}_{L R}\right)
\end{aligned}
$$

i.e., $\operatorname{per}\left(\hat{A}_{L R}\right) \leq \operatorname{per}\left(\hat{B}_{L R}\right)$. 
When $m>n$,

$$
\begin{aligned}
\operatorname{per}(\hat{A}) & =\sum_{q \in S} \prod_{j=1}^{m} \tilde{a}_{h L R q(j) j} \\
& =\sum_{q \in S} \prod_{j=1}^{m}\left(m_{q(j) j}, n_{q(j) j}, \alpha_{1 q(j) j}, \alpha_{2 q(j) j}, \beta_{i q(j) j}, \beta_{2 q(j) j}\right)_{L R} \\
& \leq \sum_{q \in S} \prod_{j=1}^{m}\left(p_{q(j) j}, q_{q(j) j}, \mu_{1 q(j) j}, \mu_{2 q(j) j}, \gamma_{1 q(j) j}, \gamma_{2 q(j) j}\right)_{L R} \\
& \leq \sum_{q \in S} \prod_{j=1}^{m} \tilde{b}_{h L R q(j) j} \\
& \leq \operatorname{per}\left(\hat{B}_{L R}\right),
\end{aligned}
$$

i.e., $\operatorname{per}\left(\hat{A}_{L R}\right) \leq \operatorname{per}\left(\hat{B}_{L R}\right)$.

Hence $\hat{A}_{L R} \leq \hat{B}_{L R} \Rightarrow \operatorname{per}\left(\hat{A}_{L R}\right) \leq \operatorname{per}\left(\hat{B}_{L R}\right)$.

Property 6.4. If any two rows (or columns) of a non-square L-R HFM $\hat{A}_{L R}$ are interchanged, then the permanent value remains unchanged.

Proof. Let $\hat{A}_{L R}=\left(\tilde{a}_{h L R i j}\right)_{m \times n}$ be a non-square L-R HFM of order $m \times n$ and $\hat{B}_{L R}=\left(\tilde{b}_{h L R i j}\right)_{m \times n}$ be the non-square L-R HFM obtain from $\hat{A}_{L R}$ by interchanging the $r$ th and $s$ th rows $(r<s)$ of $\hat{A}_{L R}$. Then, $\tilde{b}_{h L R i j}=\tilde{a}_{h L R i j}, i \neq r, j \neq s$ and $\tilde{b}_{h L R r j}=$ $\tilde{a}_{h L R s j}$ and $\tilde{b}_{h L R s j}=\tilde{a}_{h L R r j}$.

When $m \leq n$,

$$
\begin{aligned}
\operatorname{per}\left(\hat{B}_{L R}\right) & =\sum_{q \in S} \prod_{i=1}^{m} \tilde{b}_{h L R i q}(i) \\
& =\sum_{q \in S} \tilde{b}_{h L R 1 q(1)} \cdot \tilde{b}_{h L R 2 q(2)} \cdots \tilde{b}_{h L R r q(r)} \cdots \tilde{b}_{h L R s q(s)} \cdots \tilde{b}_{h L R m q(m)}
\end{aligned}
$$




$$
\begin{aligned}
= & \sum_{q \in S} \tilde{a}_{h L R 1 q(1)} \cdot \tilde{a}_{h L R 2 q(2)} \cdots \tilde{a}_{h L R s q(s)} \cdots \tilde{a}_{h L R r q(r)} \cdots \tilde{a}_{h L R m q(m)} \\
= & \sum_{q \in S}\left[\left(m_{1 q(1)}, n_{1 q(1)}, \alpha_{11 q(1)}, \alpha_{21 q(1)}, \beta_{11 q(1)}, \beta_{21 q(1)}\right)\right. \\
& \cdots\left(m_{s q(s) 1}, n_{s q(s)}, \alpha_{1 s q(s)}, \alpha_{2 s q(s)}, \beta_{1 s q(s)}, \beta_{2 s q(s)}\right) \\
& \cdots\left(m_{r q(r)}, n_{r q(r)}, \alpha_{1 r q(r)}, \alpha_{2 r q(r)}, \beta_{1 r q(r)}, \beta_{2 r q(r)}\right) \\
& \left.\cdots\left(m_{m q(m)}, n_{m q(m)}, \alpha_{1 m q(m)}, \alpha_{2 m q(m)}, \beta_{1 m q(m)}, \beta_{2 m q(m)}\right)\right] \\
= & \sum_{q \in S} \prod_{i=1}^{m} \tilde{a}_{h L R i q(i)} \\
= & \operatorname{per}\left(\hat{A}_{L R}\right) .
\end{aligned}
$$

For $m>n$, the proof is similar as before.

Hence, $\operatorname{per}\left(\hat{B}_{L R}\right)=\operatorname{per}\left(\hat{A}_{L R}\right)$.

Property 6.5. Let $\hat{A}_{L R}$ be a non-square $L-R$ HFM of order $m \times n$. If a row is multiplied by scalar $k$, then the permanent value is $k \operatorname{per}\left(\hat{A}_{L R}\right)$.

Proof. Let $\hat{A}_{L R}=\left(\tilde{a}_{h L R i j}\right)_{m \times n}$ be non-square L-R HFM of order $m \times n$ and $\hat{B}_{L R}=\left(\tilde{b}_{h L R i j}\right)_{m \times n}$ be another non-square L-R HFM obtained by multiplying $k$ to a row of $\hat{A}_{L R}$.

Case (i): If $k=0$, then the result is obviously true, since $\operatorname{per}\left(\hat{A}_{L R}\right)=0_{L R}$, when $\hat{A}_{L R}$ has a zero row

Case (ii): Let $\hat{B}_{L R}=\left(\tilde{b}_{h L R i j}\right)_{m n}$, where $\tilde{b}_{h L R i j}=\left(p_{i j}, q_{i j}, \mu_{1 i j}, \mu_{2 i j}, \gamma_{1 i j}, \gamma_{2 i j}\right)_{L R}$ is obtained from L-R HFM $\hat{A}_{L R}=\left(\tilde{a}_{h L R i j}\right)$ by multiplying its rth row by scalar $k \neq 0$.

Obviously $\left(p_{i j}, q_{i j}, \mu_{1 i j}, \mu_{2 i j}, \gamma_{1 i j}, \gamma_{2 i j}\right)_{L R}=\left(m_{i j}, n_{i j}, \alpha_{1 i j}, \alpha_{2 i j}, \beta_{1 i j}, \beta_{2 i j}\right)_{L R}$ for all $i \neq 0$. 
When $k>0$, we have

$$
\left(p_{i j}, q_{i j}, \mu_{1 i j}, \mu_{2 i j}, \gamma_{1 i j}, \gamma_{2 i j}\right)_{L R}=\left(k m_{i j}, k n_{i j}, k \alpha_{1 i j}, k \alpha_{2 i j}, k \beta_{1 i j}, k \beta_{2 i j}\right)_{L R}
$$

When $k<0$, we have

$$
\left(p_{i j}, q_{i j}, \mu_{1 i j}, \mu_{2 i j}, \gamma_{1 i j}, \gamma_{2 i j}\right)_{L R}=\left(k n_{i j}, k m_{i j}, k \alpha_{2 i j}, k \alpha_{1 i j}, k \beta_{2 i j}, k \beta_{1 i j}\right)_{L R} .
$$

Then by the definition:

When $m \leq n$, then

$$
\begin{aligned}
\operatorname{per}\left(\hat{B}_{L R}\right)= & \sum_{q \in S} \prod_{i=1}^{m}\left(\tilde{b}_{h L R i q(i)}\right) \\
= & \sum_{q \in S} \prod_{i=1}^{m} \tilde{b}_{h L R 1 q(1)} \cdot \tilde{b}_{h L R 2 q(2)} \cdots \tilde{b}_{h L R m q(m)} \\
= & \sum_{q \in S}\left[\left(p_{1 q(1)}, q_{1 q(1)}, \mu_{11 q(1)}, \mu_{21 q(1)}, \gamma_{11 q(1)}, \gamma_{21 q(1)}\right)_{L R}\right. \\
& \cdots\left(p_{r q(r)}, q_{r q(r)}, \mu_{1 r q(r)}, \mu_{2 r q(r)}, \gamma_{1 r q(r)}, \gamma_{2 r q(r)}\right)_{L R} \\
& \left.\cdots\left(p_{m q(m)}, q_{m q(m)}, \mu_{1 m q(m)}, \mu_{2 m q(m)}, \gamma_{1 m q(m)}, \gamma_{2 m q(m)}\right)_{L R}\right]
\end{aligned}
$$

When $k>0$,

$$
\begin{aligned}
\operatorname{per}\left(\hat{B}_{L R}\right)= & \sum_{q \in S}\left[\left(p_{1 q(1)}, q_{1 q(1)}, \mu_{11 q(1)}, \mu_{21 q(1)}, \gamma_{11 q(1)}, \gamma_{21 q(1)}\right)_{L R}\right. \\
& \cdots\left(p_{r q(r)}, q_{r q(r)}, \mu_{1 r q(r)}, \mu_{2 r q(r)}, \gamma_{1 r q(r)}, \gamma_{2 r q(r)}\right)_{L R} \\
& \left.\cdots\left(p_{m q(m)}, q_{m q(m)}, \mu_{1 m q(m)}, \mu_{2 m q(m)}, \gamma_{1 m q(m)}, \gamma_{2 m q(m)}\right)_{L R}\right] \\
= & k \sum_{q \in s}\left[\left(m_{1 q(1)}, n_{1 q(1)}, \alpha_{11 q(1)}, \alpha_{21 q(1)}, \beta_{11 q(1)}, \beta_{21 q(1)}\right)_{L R}\right. \\
& \cdots\left(m_{r q(r)}, n_{r q(r)}, \alpha_{1 r q(r)}, \alpha_{2 r q(r)}, \beta_{1 r q(r)}, \beta_{2 r q(r)}\right)_{L R} \\
& \left.\cdots\left(m_{m q(m)}, n_{m q(m)}, \alpha_{1 m q(m)}, \alpha_{2 m q(m)}, \beta_{1 m q(m)}, \beta_{2 m q(m)}\right)_{L R}\right]
\end{aligned}
$$




$$
\begin{aligned}
& =k \sum_{q \in S} \prod_{i=1}^{m}\left(\tilde{a}_{h L R i q(i)}\right) \\
& =\operatorname{kper}\left(\hat{A}_{L R}\right) .
\end{aligned}
$$

When $k<0$,

$$
\begin{aligned}
& \operatorname{per}\left(\hat{B}_{L R}\right)=\sum_{q \in S}\left[\left(p_{1 q(1)}, q_{1 q(1)}, \mu_{11 q(1)}, \mu_{21 q(1)}, \gamma_{11 q(1)}, \gamma_{21 q(1)}\right)_{L R}\right. \\
& \cdots\left(p_{r q(r)}, q_{r q(r)}, \mu_{1 r q(r)}, \mu_{2 r q(r)}, \gamma_{1 r q(r)}, \gamma_{2 r q(r)}\right)_{L R} \\
& \left.\cdots\left(p_{m q(m)}, q_{m q(m)}, \mu_{1 m q(m)}, \mu_{2 m q(m)}, \gamma_{1 m q(m)}, \gamma_{2 m q(m)}\right)_{L R}\right] \\
& =\sum_{q \in S}\left[\left(m_{1 q(1)}, n_{1 q(1)}, \alpha_{11 q(1)}, \alpha_{21 q(1)}, \beta_{11 q(1)}, \beta_{21 q(1)}\right)_{L R}\right. \\
& \cdots\left(k n_{r q(r)}, k m_{r q(r)}, k \alpha_{2 r q(r)}, k \alpha_{1 r q(r)}, k \beta_{2 r q(r)}, k \beta_{1 r q(r)}\right)_{L R} \\
& \left.\cdots\left(m_{m q(m)}, n_{m q(m)}, \alpha_{1 m q(m)}, \alpha_{2 m q(m)}, \beta_{1 m q(m)}, \beta_{2 m q(m)}\right)_{L R}\right] \\
& =k \sum_{q \in S}\left[\left(m_{1 q(1)}, n_{1 q(1)}, \alpha_{11 q(1)}, \alpha_{21 q(1)}, \beta_{11 q(1)}, \beta_{21 q(1)}\right)_{L R}\right. \\
& \cdots\left(m_{r q(r)}, n_{r q(r)}, \alpha_{1 r q(r)}, \alpha_{2 r q(r)}, \beta_{1 r q(r)}, \beta_{2 r q(r)}\right)_{L R} \\
& \left.\cdots\left(m_{m q(m)}, n_{m q(m)}, \alpha_{1 m q(m)}, \alpha_{2 m q(m)}, \beta_{1 m q(m)}, \beta_{2 m q(m)}\right)_{L R}\right] \\
& =k \sum_{q \in s} \prod_{i=1}^{m}\left(\tilde{a}_{h \operatorname{LRiq}(i)}\right) \\
& =\operatorname{kper}\left(\hat{A}_{L R}\right) \text {. }
\end{aligned}
$$

i.e., $\operatorname{per}\left(\hat{A}_{L R}\right)=\operatorname{kper}\left(\hat{A}_{L R}\right)$.

For $m>n$, the proof is similar as before.

Hence $\operatorname{per}\left(\hat{A}_{L R}\right)=\operatorname{kper}\left(\hat{A}_{L R}\right)$.

Property 6.6. For any non-square hexagonal fuzzy matrix $(H F M) \hat{A}_{L R}=\left(\tilde{a}_{h L R i j}\right)$ of order $m \times n, \operatorname{per}\left(\hat{A}_{L R}\right)=\operatorname{per}\left(\hat{A}_{L R}^{T}\right)$. 
Proof. Let $\hat{A}_{L R}=\left(\tilde{a}_{h L R i j}\right)_{m \times n}$ and $\tilde{a}_{h L R i j}=\left(m_{i j}, n_{i j}, \alpha_{1 i j}, \alpha_{2 i j}, \beta_{1 i j}, \beta_{2 i j}\right)_{L R}$.

When $m \leq n$,

$$
\operatorname{per}\left(\hat{A}_{L R}\right)=\sum_{q \in S} \prod_{i=1}^{m}\left(\tilde{a}_{h L R i q(i)}\right)
$$

Let $\hat{A}_{L R}^{T}=\hat{B}_{L R}=\left(\tilde{b}_{h L R i j}\right)_{m n}, n \geq m$. Then, $\tilde{b}_{h L R i j}=\tilde{a}_{h L R i j}$,

$$
\begin{aligned}
\operatorname{per}\left(\hat{A}_{L R}^{T}\right) & =\operatorname{per}\left(\hat{B}_{L R}\right) \\
& =\sum_{q \in S} \prod_{j=1}^{m}\left(\tilde{b}_{h L R q(j) j}\right) \\
& =\sum_{q \in S} \prod_{j=1}^{m}\left(\tilde{a}_{h L R q(j) j}\right) \\
& =\sum_{q \in S} \prod_{i=1}^{m}\left(\tilde{a}_{h L R i q(i)}\right) \\
& =\operatorname{per}\left(\hat{A}_{L R}\right) \\
\operatorname{per}\left(\hat{A}_{L R}^{T}\right) & =\operatorname{per}\left(\hat{A}_{L R}\right) .
\end{aligned}
$$

For $m>n$,

$$
\begin{aligned}
\operatorname{per}\left(\hat{A}_{L R}^{T}\right) & =\operatorname{per}\left(\hat{B}_{L R}\right) \\
& =\sum_{q \in S} \prod_{i=1}^{n}\left(\tilde{b}_{h L R i q(i)}\right) \\
& =\sum_{q \in S} \prod_{i=1}^{n}\left(\tilde{a}_{h L R q(i) i}\right) \\
& =\sum_{q \in S} \prod_{j=1}^{m}\left(\tilde{a}_{h L R i q(i)}\right)
\end{aligned}
$$




$$
\begin{aligned}
& =\operatorname{per}\left(\hat{A}_{L R}\right) \\
\operatorname{per}\left(\hat{A}_{L R}^{T}\right) & =\operatorname{per}\left(\hat{A}_{L R}\right) .
\end{aligned}
$$

Hence $\operatorname{per}\left(\hat{A}_{L R}^{T}\right)=\operatorname{per}\left(\hat{A}_{L R}\right)$.

\subsection{Non-square constant L-R HFM}

In this section, we have to define the notion of non-square constant L-R HFM. Some important properties are proved by the permanent notion with suitable examples.

Definition 6.7. Non-Square R-Constant L-R HFM

An L-R HFM $\hat{A}_{L R}=\left(\tilde{a}_{h L R i j}\right)$ of order $m \times n$ is called a non-square $R$-constant $L-R$ $H F M$ if all its rows are equal to each other. That is, $\tilde{a}_{h L R i j}=\tilde{a}_{h L R r j}$ for all $i, r=1,2, \ldots, m$ and $j=1,2, \ldots, n$.

\section{Definition 6.8. Non-Square C-Constant L-R HFM}

An L-R HFM $\hat{A}_{L R}=\left(\tilde{a}_{h L R i j}\right)$ of order $m \times n$ is called a non-square C-constant $L-R$ $H F M$ if all its columns are equal to each other. That is, $\tilde{a}_{h L R i j}=\tilde{a}_{h L R i r}$ for all $i=1,2, \ldots, m$ and $r, j=1,2, \ldots, n$.

\section{Definition 6.9. Non-Square Constant L-R HFM}

An L-R HFM $\hat{A}=\left(\tilde{a}_{h L R i j}\right)$ of order $m \times n$ is called a non-square constant $L-R$ $H F M$ if it is either non-square R-Constant L-R HFM or non-square C-Constant L-R HFM.

For Example: Let

$$
\hat{A}_{L R}=\left(\begin{array}{ccc}
(1,2,1,1,2,2) & (6,8,2,2,2,2) & (3,5,1,1,1,1) \\
(1,2,1,1,1,1) & (6,8,2,2,2,2) & (3,5,1,1,1,1)
\end{array}\right)
$$

be non-square R-constant LR HFM and let

$$
\hat{B}_{L R}=\left(\begin{array}{cc}
(1,2,1,1,2,2) & (1,2,1,1,2,2) \\
(6,8,2,2,2,2) & (6,8,2,2,2,2) \\
(3,5,1,1,1,1) & (3,5,1,1,1,1)
\end{array}\right)
$$

be non-square C-constant L-R HFM. 


\subsection{Properties of non-square constant L-R HFM}

Property 6.10. Let $\hat{A}_{L R}$ and $\hat{B}_{L R}$ be two non-square constant L-R HFMs of same order. Then

$$
\hat{A}_{L R}+\hat{B}_{L R}=\hat{B}_{L R}+\hat{A}_{L R} \Rightarrow \operatorname{per}\left(\hat{A}_{L R}+\hat{B}_{L R}\right)=\operatorname{per}\left(\hat{B}_{L R}+\hat{A}_{L R}\right) .
$$

Proof. Let $\hat{A}_{L R}=\left(\tilde{a}_{h L R i j}\right)_{m \times n}$ and $\hat{B}_{L R}=\left(\tilde{b}_{h L R i j}\right)_{m \times n}$, where $\tilde{a}_{h L R i j}=\left(m_{i j}, n_{i j}\right.$, $\left.\alpha_{1 i j}, \alpha_{2 i j}, \beta_{1 i j}, \beta_{2 i j}\right)_{L R}$ and $\tilde{b}_{h L R i j}=\left(p_{i j}, q_{i j}, \mu_{1 i j}, \mu_{2 i j}, \gamma_{1 i j}, \gamma_{2 i j}\right)_{L R}$ are two nonsquare constant L-R HFMs of order $m \times n$. Then $\tilde{a}_{h L R i j}=\tilde{a}_{h L R r j}$ and $\tilde{b}_{h L R i j}=\tilde{b}_{h L R r j}$.

Let $\hat{C}_{L R}=\left(\tilde{c}_{h L R i j}\right)=\left(\tilde{a}_{h L R i j}+\tilde{b}_{h L R i j}\right)=\left(\tilde{a}_{h L R r j}+\tilde{b}_{h L R r j}\right)=\tilde{c}_{h L R r j}$, for all $i, r=$ $1,2, \ldots, m$ and $j=1,2, \ldots, n$.

Let $\hat{D}_{L R}=\left(\tilde{d}_{h L R i j}\right)=\left(\tilde{b}_{h L R i j}+\tilde{a}_{h L R i j}\right)=\left(\tilde{b}_{h L R r j}+\tilde{a}_{h L R r j}\right)=\tilde{d}_{h L R r j}$, for all $i, r=$ $1,2, \ldots, m$ and $j=1,2, \ldots, n$.

When $m \leq n$,

$$
\begin{aligned}
\operatorname{per}\left(\hat{A}_{L R}+\hat{B}_{L R}\right)= & \operatorname{per}\left(\hat{C}_{L R}\right) \\
= & \sum_{q \in S} \prod_{i=1}^{m} \tilde{c}_{h L R i q(i)} \\
= & \sum_{q \in S}\left(\tilde{c}_{h L R 1 q(1)}\right) \cdot\left(\tilde{c}_{h L R 2 q(2)}\right) \cdots\left(\tilde{c}_{h L R m q(m)}\right) \\
= & \sum_{q \in S}\left(\tilde{a}_{h L R 1 q(1)}+\tilde{b}_{h L R 1 q(1)}\right) \cdot\left(\tilde{a}_{h L R 2 q(2)}+\tilde{b}_{h L R 2 q(2)}\right) \\
& \cdots\left(\tilde{a}_{h L R m q(m)}+\tilde{b}_{h L R m q(m)}\right) \\
= & \sum_{q \in S}\left(\tilde{a}_{h L R r q(r)}+\tilde{b}_{h L R r q(r)}\right) \cdot\left(\tilde{a}_{h L R r q(r)}+\tilde{b}_{h L R r q(r)}\right) \\
& \cdots\left(\tilde{a}_{h L R r q(r)}+\tilde{b}_{h L R r q(r)}\right)
\end{aligned}
$$




$$
\begin{aligned}
= & \sum_{q \in S}\left(\tilde{b}_{h L R r q(r)}+\tilde{a}_{h L R r q(r)}\right) \cdot\left(\tilde{b}_{h L R r q(r)}+\tilde{a}_{h L R r q(r)}\right) \\
& \cdots\left(\tilde{b}_{h L R r q(r)}+\tilde{a}_{h L R r q(r)}\right) \\
= & \sum_{q \in S} \prod_{r=1}^{m}\left(\tilde{b}_{h L R r q(r)}+\tilde{a}_{h L R r q(r)}\right) \\
= & \sum_{q \in S} \prod_{r=1}^{m} \tilde{d}_{h L R i q(i)} \\
= & \operatorname{per}\left(\hat{D}_{L R}\right) \\
= & \operatorname{per}\left(\hat{B}_{L R}+\hat{A}_{L R}\right)
\end{aligned}
$$

i.e., $\operatorname{per}\left(\hat{A}_{L R}+\hat{B}_{L R}\right)=\operatorname{per}\left(\hat{B}_{L R}+\hat{A}_{L R}\right)$.

For $m>n$, the proof is similar as before.

Hence $\operatorname{per}\left(\hat{A}_{L R}+\hat{B}_{L R}\right)=\operatorname{per}\left(\hat{B}_{L R}+\hat{A}_{L R}\right)$.

Property 6.11. Let $\hat{A}_{L R}$ and $\hat{B}_{L R}$ be two non-square constant L-R HFMs of same order. Then

$$
\hat{A}_{L R} \cdot \hat{B}_{L R}^{T}=\hat{B}_{L R} \cdot \hat{A}_{L R}^{T} \Rightarrow \operatorname{per}\left(\hat{A}_{L R} \cdot \hat{B}_{L R}^{T}\right)=\operatorname{per}\left(\hat{B}_{L R} \cdot \hat{A}_{L R}^{T}\right)
$$

Proof. Let $\hat{A}_{L R}=\left(\tilde{a}_{h L R i j}\right)_{m \times n}$ and $\hat{A}_{L R}=\left(\tilde{a}_{h L R i j}\right)_{m \times n}$. Then $\hat{A}_{L R}^{T}=\hat{C}_{L R}=$ $\left(\tilde{c}_{h L R i j}\right)_{m \times n}$ and $\hat{B}_{L R}^{T}=\hat{D}_{L R}=\left(\tilde{d}_{h L R i j}\right)_{m \times n}$. That is, all the non-square constant L-R HFMs are

$$
\begin{aligned}
& \tilde{a}_{h L R i j}=\tilde{a}_{h L R r j} ; \tilde{b}_{h L R i j}=\tilde{b}_{h L R r j} \text { for all } i, r=1,2, \ldots, m \text { and } j=1,2, \ldots, n, \\
& \tilde{c}_{h L R i j}=\tilde{c}_{h L R i r} ; \tilde{d}_{h L R i j}=\tilde{d}_{h L R i r} \text { for all } i=1,2, \ldots, m \text { and } r, j=1,2, \ldots, n .
\end{aligned}
$$

Let

$$
\hat{A}_{L R} \cdot \hat{B}_{L R}^{T}=\hat{X}_{L R}=\tilde{x}_{h L R i j}
$$




$$
\begin{aligned}
& =\sum_{p=1}^{n} \tilde{a}_{h L R i p} \cdot \tilde{d}_{h L R p j} \\
& =\sum_{p=1}^{n} \tilde{a}_{h L R r p} \cdot \tilde{d}_{h L R p r}, i, r, p, j=1,2, \ldots, n \\
\hat{B}_{L R} \cdot \hat{A}_{L R}^{T}= & \hat{Y}_{L R}=\tilde{y}_{h L R i j} \\
= & \sum_{p=1}^{n} \tilde{b}_{h L R i p} \cdot \tilde{c}_{h L R p j} \\
= & \sum_{p=1}^{n} \tilde{b}_{h L R r p} \cdot \tilde{c}_{h L R p r}, \quad i, r, p, j=1,2, \ldots, n .
\end{aligned}
$$

Let

$$
\begin{aligned}
\hat{A}_{L R} \cdot \hat{B}_{L R}^{T} & =\hat{X}_{L R}=\left(\tilde{x}_{h L R i j}\right) \\
& =\sum_{p=1}^{n}\left(\tilde{a}_{h L R i p} \cdot \tilde{d}_{h L R p j}\right) \\
& =\sum_{p=1}^{n}\left(\tilde{a}_{h L R i r} \cdot \tilde{d}_{h L R r j}\right), i, r, p, j=1,2, \ldots, n . \\
\hat{B}_{L R} \cdot \hat{A}_{L R}^{T} & =\hat{Y}_{L R}=\left(\tilde{y}_{h L R i j}\right) \\
& =\sum_{p=1}^{n}\left(\tilde{b}_{h L R i p} \cdot \tilde{c}_{h L R p j}\right) \\
& =\sum_{p=1}^{n}\left(\tilde{b}_{h L R i r} \cdot \tilde{c}_{h L R r j}\right), i, r, p, j=1,2, \ldots, n .
\end{aligned}
$$

When $m \leq n$,

$$
\begin{aligned}
\operatorname{per}\left(\hat{A}_{L R} \cdot \hat{B}_{L R}^{T}\right) & =\operatorname{per}\left(\hat{X}_{L R}\right) \\
& =\sum_{q \in S} \prod_{i=1}^{m} \tilde{x}_{h L R i q(i)}
\end{aligned}
$$




$$
\begin{aligned}
& =\sum_{q \in S} \prod_{i=1}^{m} \sum_{p=1}^{n} \tilde{a}_{h L R i p} \cdot \tilde{d}_{h L R p j} \\
& =\sum_{q \in S} \prod_{i=1}^{m} \sum_{p=1}^{n} \tilde{a}_{h L R r p} \cdot \tilde{d}_{h L R p r} \\
& =\sum_{q \in S} \prod_{i=1}^{m} \sum_{p=1}^{n} \tilde{b}_{h L R r p} \cdot \tilde{c}_{h L R p r} \\
& =\sum_{q \in S} \prod_{i=1}^{m} \tilde{y}_{h L R i q(i)} \\
& =\operatorname{per}\left(\hat{Y}_{L R}\right) \\
& =\operatorname{per}\left(\hat{B}_{L R} \cdot \hat{A}_{L R}^{T}\right) .
\end{aligned}
$$

i.e., $\operatorname{per}\left(\hat{A}_{L R} \cdot \hat{B}_{L R}^{T}\right)=\operatorname{per}\left(\hat{B}_{L R} \cdot \hat{A}_{L R}^{T}\right)$.

For $m>n$, the proof is similar as before.

Hence $\operatorname{per}\left(\hat{A}_{L R} \cdot \hat{B}_{L R}^{T}\right)=\operatorname{per}\left(\hat{B}_{L R} \cdot \hat{A}_{L R}^{T}\right)$.

\section{Conclusion}

In this work, it is a notion which is ambiguously related to a determinant so, we follow closely the approach and introduce for the determinant. We have also studied the notion called the permanent of both square and non-square L-R HFM, which contains some of the theorems and standard properties which are verified. The fuzzy permanent matrices can be developed using eigenvalue properties in future.

\section{References}

[1] A. Das, M. Pal and M. Bhowmik, Permanent of interval-valued and triangular number fuzzy matrices, Ann. Fuzzy Math. Inform. 10(3) (2015), 381-395.

[2] D. Dubois and H. Prade, Fuzzy Sets and Systems. Theory and Applications, London: Academic Press, 1980. 
[3] D.G. Glynn, The permanent of a square matrix, European J. Combin. 31 (2010), 18871891.

[4] Liu Wang-jin and Yi Liang-Zhong, The permanent of L-fuzzy matrices and some applications, Fuzzy Sets and Systems, 1990.

[5] Natália Bebiano, On the evaluation of permanents, Pacific J. Math. 101(1) (1982), 1-9.

[6] E. Romanwicz and A. Grabowski, On the permanent of a matrix, Formalized Mathematics 14(1) (2006), 13-20.

[7] H.J. Ryser and R.A. Brualdi, Combinatorial matrix theory, Encyclopedia of Mathematics and its Applications, 39, Cambridge: Cambridge University Press, 1991.

[8] A.K. Shyamal and M. Pal, Triangular fuzzy matrices, Iran. J. Fuzzy Syst. 4(1) (2007), 7587.

[9] D. Stephen Dinagar and K. Latha, On constant type-2 triangular fuzzy matrices, International Journal of Applications of Fuzzy sets and Artificial Intelligence 4 (2014), 215-226.

[10] D. Stephen Dinagar and J. Rajesh Kannan, On inventory model with allowable shortage using LR-type hexagonal fuzzy numbers, International Journal of Applications of Fuzzy Sets and Artificial Intelligence 6 (2016), 215-226.

[11] D. Stephen Dinagar and U. Hari Narayanan, On inverse of hexagonal fuzzy number matrices, Int. J. Pure Appl. Math. 115(9) (2017), 147-158.

[12] D. Stephen Dinagar and U. Hari Narayanan, A study on orthogonal fuzzy number matrices, Global J. Pure Appl. Math. 13(2) (2017), 1-11.

[13] M.G. Thomason, Convergence of powers of a fuzzy matrix, J. Math. Anal. Appl. 57 (1977), 476-480.

[14] L.A. Zadeh, Fuzzy sets, Information and Control 8 (1965), 338-353. 